\title{
Assessment of the Biomechanical Properties of the Cornea with the Ocular Response Analyzer in Normal and Keratoconic Eyes
}

\author{
Sunil Shab, ${ }^{1,2,3}$ Mobammed Laiquzzaman, ${ }^{1}$ Rajan Bbojwani, ${ }^{3}$ Sanjay Mantry, ${ }^{1,3}$ \\ and Ian Cunliffe (1,2,3 $^{1}$
}

Purpose. To compare hysteresis, a novel measure of ocular rigidity (viscoelasticity) in normal and keratoconic eyes.

Methods. The study consisted of 207 normal and 93 keratoconic eyes. Eyes were diagnosed as keratoconic based on clinical examination and corneal topography. The hysteresis was measured by the Ocular Response Analyzer (ORA; Reichert Ophthalmic Instruments, Buffalo, NY). The data were recorded by Generation 3 software for the ORA. Central corneal thickness (CCT) was measured with a handheld ultrasonic pachymeter in the midpupillary axis.

Results. The mean hysteresis was $10.7 \pm 2.0$ (SD) $\mathrm{mm} \mathrm{Hg}$ (range, 6.1-17.6) in normal eyes compared with $9.6 \pm 2.2 \mathrm{~mm}$ $\mathrm{Hg}$ (range, 4.7-16.7) in keratoconic eyes. The difference was statistically significant $(P<0.0001$, unpaired $t$-test). Mean CCT in the normal and keratoconic eyes was 545.0 $\pm 36.4 \mu \mathrm{m}$ (range, 471-650) and 491.8 $\pm 54.7 \mu \mathrm{m}$ (range, 341-611), respectively; the difference was significant $(P<0.0001$, unpaired $t$-test).

Conclusions. Hysteresis was significantly higher in normal than in keratoconic eyes. It may be a useful measurement in addition to CCT, when assessing ocular rigidity, and may be of particular importance when trying to correct intraocular measurements for increased or decreased ocular rigidity. Long-term studies of change in hysteresis may provide information on the progression of keratoconus. (Invest Ophthalmol Vis Sci. 2007; 48:3026-3031) DOI:10.1167/iovs.04-0694

K eratoconus is a noninflammatory condition of unknown etiology affecting the central cornea characterized by thinning and ectasia of the cornea. ${ }^{1}$ Increased distensibility has been reported to be an important factor in the pathogenesis of keratoconus. ${ }^{2}$ It may affect vision significantly due to irregular astigmatism and corneal scarring. Keratoconus is usually a bilateral condition, but approximately $17 \%$ of cases have been reported to be unilateral. ${ }^{3}$ Keratoconic eyes are known to be more elastic and less rigid than normal eyes and hence may have a different hysteresis than do normal eyes. One possible

From the ${ }^{1}$ Birmingham Heartlands and Solihull NHS Trust, Solihull, United Kingdom; the ${ }^{2}$ Anterior Eye Group (AEG), Neurosciences Research Institute, Aston University, Birmingham, United Kingdom; and the ${ }^{3}$ Birmingham and Midland Eye Centre, Birmingham, United Kingdom.

Submitted for publication June 14, 2004; revised August 31 and December 2, 2005, and April 23 and July 5, 2006; accepted May 21, 2007.

Disclosure: S. Shah, None; M. Laiquzzaman, None; R. Bhojwani, None; S. Mantry, None; I. Cunliffe, None

The publication costs of this article were defrayed in part by page charge payment. This article must therefore be marked "advertise$m e n t "$ in accordance with 18 U.S.C. $\$ 1734$ solely to indicate this fact.

Corresponding author: Sunil Shah, Birmingham Heartlands and Solihull NHS Trust, Lode Lane, Solihull, West Midlands, UK B91 2JL; sunilshah@doctors.net.uk. measure of ocular rigidity is hysteresis. This study was conducted to compare hysteresis in normal and keratoconic eyes using the Ocular Response Analyzer (ORA; Reichert Ophthalmic Instruments, Buffalo, NY).

So far, there is no easy method reported to determine the biomechanical properties of the cornea in vivo. Reichert has developed a new device, the ORA, which is an adaptation of their noncontact tonometer (NCT) to allow measurement of IOP as well as new metrics referred to as hysteresis, which is said to be a measure of the viscoelastic properties of the eye.

Hysteresis is a parameter to characterize the biomechanical status of the cornea. It is determined by releasing an air puff from the NCT causing inward and then outward corneal motion, which in turn provides two applanation measurements during a single measurement process (Fig. 1). Hysteresis is a measurement that is the result of the damping of the cornea because of its viscoelastic properties and is derived from the difference of the two applanation measurements during the applanation process. Thus, hysteresis is ocular resistance due to the combined effect of the parameters such as corneal thickness, ocular rigidity, and viscoelastic properties. ${ }^{4}$

\section{Materials ANd Methods}

Healthy volunteers were recruited from the staff and relatives of patients attending the ophthalmology clinic in a teaching hospital in Birmingham, United Kingdom. Two hundred seven normal eyes of 42 men and 63 women were selected. The mean age of the patients was $62.1 \pm 18.1(\mathrm{SD})$ years (range, 18.1-87.1). All patients had normal corneas (based on history and examination). None of the subjects had glaucoma, had undergone previous eye surgery, had had an eye infection, were using any topical eye medication, or had a history of generalized disease

Ninety-three keratoconic eyes from 38 males and 20 females were recruited from a specialist corneal clinic. The average age of the patients was $32.3 \pm 12.1$ years (range, 17.1-77.1 years). The diagnosis of keratoconus was made by an experienced corneal specialist (SS) on the basis of the following diagnostic criteria (one sign or a combination of signs): external signs such as Munson's sign (V-shaped conformation of the lower lid on down gaze), Rizzuti's sign (sharply focused beam on nasal limbus produced by illumination temporally); and biomicroscopic signs such as stromal thinning, conical protrusion, Fleischer's ring, Vogt's striae, and enlarged corneal nerves; topographic signs consistent with keratoconus; and an abnormal retinoscopy reflex.

In addition to the diagnosis of keratoconus, an attempt was made to grade the severity of the condition. The severity was graded as mild, moderate, and severe on the basis of Orbscan II (Bausch \& Lomb Surgical, Rochester, NY) readings. Five objective measurements were determined (anterior corneal curvatures, difference of astigmatisms in each meridian, and anterior and posterior best fit spheres). Each eye was given a score for each of the parameters (e.g., a score of 1 for anterior keratometry $\mathrm{K} 1$, if $\mathrm{K} 1$ was between 45.1 and 46.9). In addition, an overall subjective assessment of the Orbscan image was made and scored from 0 to 3 (Table 1). This is a new technique in which Orbscan II images are used in an attempt to grade the severity of 
FIGURE 1. Measurement of ocular hysteresis.

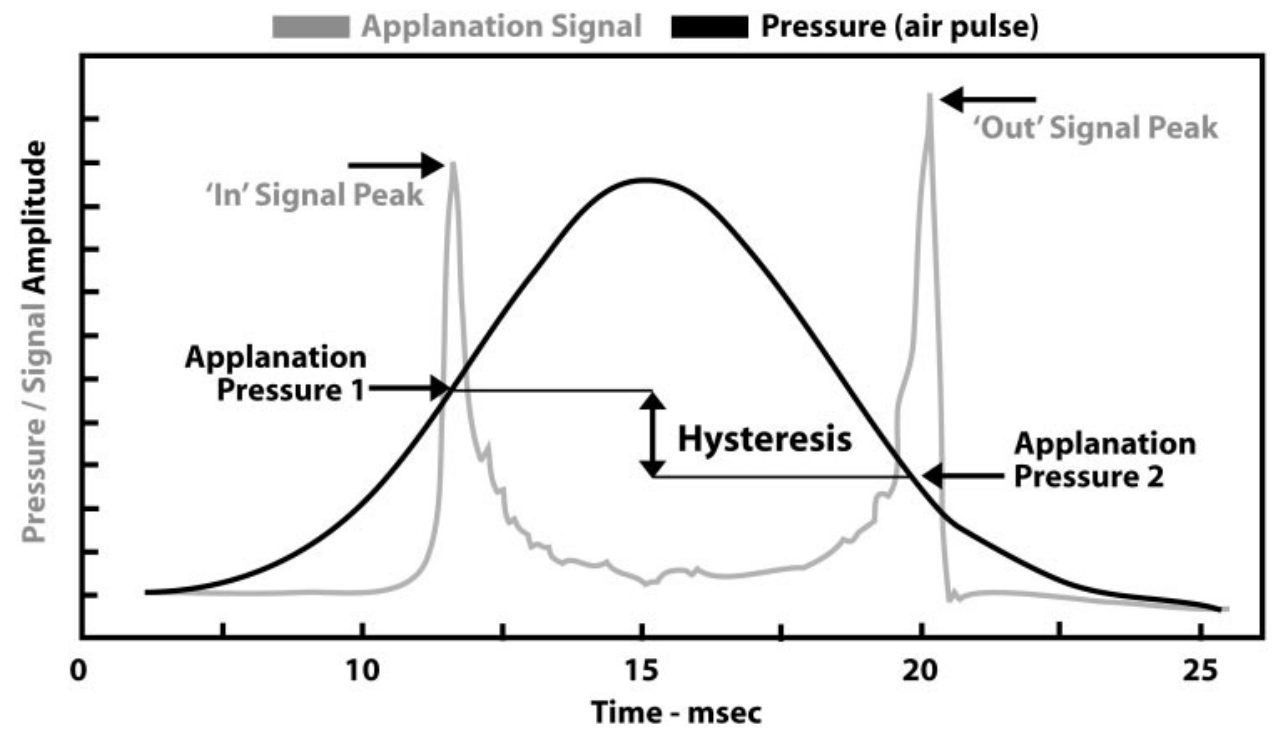

keratoconus objectively. Each parameter was graded 0 to 3, and a total score was calculated. A total score of 0 to 2 was considered normal, 3 to 6 as mild, 7 to 11 as moderate, and more than 12 as severe. The clinical grading and criteria were performed by an experienced corneal surgeon (SS).

Hysteresis is measured with the ORA while the subject is sitting in a chair. The patient is asked to fixate at the target (red blinking light) in the ORA, and the ORA is activated by pressing a button attached to the computer. A noncontact probe scans the central area of the eye, releases an air puff, and sends a signal to the ORA. The ORA then displays the hysteresis on the monitor of the computer attached to the ORA. The data are collected by the Generation 3 software for the ORA (Reichert Ophthalmic Instruments). The CCT was measured with a handheld ultrasonic pachymeter (DGH-550; DGH Technology Inc., Exton, PA) while the patient was seated. one drop of the topical anesthetic proxymetacaine (Bausch \& Lomb) was instilled in both eyes. The patient was asked to fixate a target to minimize eye movement and to avoid damage to the corneal epithelium. The pachymeter probe was gently placed on the midpupillary axis in a perpendicular orientation. On contact with the corneal surface, the CCT was displayed on the monitor attached to the probe. Three readings were taken, and the mean was used as the CCT.

The study and data accumulation were performed with the approval of the local ethics committee, informed consent was obtained from each subject participating in the study, and the study protocol was consistent with the tenets of the Declaration of Helsinki.

\section{Statistical Analysis of Data}

Several computer packages were used to analyze and present the data obtained (Excel; Microsoft Corp., Redmond, WA; and MedCalc; MedCalc Software, Mariakerke, Belgium). The overall statistical approach was based on standard texts. ${ }^{5}$

For general statistical reporting, the mean values from each data set were calculated along with the standard deviation. The distributions of values within each data set were evaluated graphically. The level of statistical significance was set at $P<0.05$. All graphs were constructed using the software programs just mentioned.

\section{Results}

The mean hysteresis was $10.7 \pm 2.0$ (SD) $\mathrm{mm} \mathrm{Hg}$ (range, 6.1-17.6) in normal eyes compared with $9.6 \pm 2.2 \mathrm{~mm} \mathrm{Hg}$ (range, 4.7-16.7) in keratoconic eyes. The difference was statistically significant $(P<0.0001$, unpaired $t$-test). The CCT was $545.0 \pm 36.4 \mu \mathrm{m}$ in normal eyes and $491.8 \pm 54.7 \mu \mathrm{m}$ in the keratoconic eyes, the difference was statistically significant $(P<0.0001$, unpaired $t$-test; Table 2$)$.

Figure 2 shows the frequency of distribution of hysteresis in normal and keratoconic eyes. Figures 3 and 4 are box-andwhisker plots showing the median and interquartile ranges of hysteresis and CCT of the normal and keratoconic eyes, respectively. Figures 5 and 6 show the relationship (scatterplot) between hysteresis and CCT of normal and keratoconic eyes. The slope of the simple regression line was moderate for all keratoconic eyes (correlation coefficient $r=0.45$ ) but the relationship was significant $(P<0.0001)$. The correlation coefficient for the normal eyes was 0.42 -a significant relationship $(P<0.0001)$.

The regression equation for normal eyes was

$$
\text { Hysteresis }=0.023 C C T-1.776
$$

whereas that for keratoconic eyes was

$$
\text { Hysteresis }=0.018 C C T+0.681
$$

TABLE 1. The Criteria for Grading of Severity of Keratoconic Eyes

\begin{tabular}{ccccccc}
\hline $\begin{array}{c}\text { Grading } \\
\text { Score }\end{array}$ & $\begin{array}{c}\text { Anterior } \\
\text { Keratometry } \\
\text { K1 }\end{array}$ & $\begin{array}{c}\text { Anterior } \\
\text { Keratometry } \\
\text { K2 }\end{array}$ & $\begin{array}{c}\text { Difference } \\
\text { of } \\
\text { Sim K }\end{array}$ & $\begin{array}{c}\text { Anterior } \\
\text { Best Fit } \\
\text { Sphere }\end{array}$ & $\begin{array}{c}\text { Posterior } \\
\text { Best Fit } \\
\text { Sphere }\end{array}$ & $\begin{array}{c}\text { Orbscan } \\
\text { Image } \\
\text { Grading }\end{array}$ \\
\hline 0 & $<45.0$ & $<45.0$ & $0-1.49$ & $0-41.9$ & $0-50.0$ & 0 \\
1 & $45.1-46.9$ & $45.1-46.9$ & $1.5-2.49$ & $42.1-44.0$ & $50.1-52.0$ & 1 \\
2 & $47.0-49.9$ & $47.0-49.9$ & $2.5-3.45$ & $44.1-46.0$ & $52.1-56.0$ & 2 \\
3 & $>50.0$ & $>50.0$ & $>3.5$ & $>46.1$ & $>56.1$ & 3 \\
\hline
\end{tabular}


TABLE 2. Hysteresis and CCT of Keratoconic Eyes According to Grading

\begin{tabular}{lcccc}
\hline & $\begin{array}{c}\text { Hysteresis } \\
(\mathbf{m m ~ H g})\end{array}$ & $\begin{array}{c}\text { Range } \\
(\mathbf{m m ~ H g})\end{array}$ & $\begin{array}{c}\text { CCT } \\
(\boldsymbol{\mu m})\end{array}$ & $\begin{array}{c}\text { Range } \\
(\boldsymbol{\mu m})\end{array}$ \\
\hline Normal $(n=207)$ & $10.7 \pm 2.0$ & $6.1-17.6$ & $545.0 \pm 36.4$ & $471-650$ \\
Keratoconus $(n=93$ eyes $)$ & $9.6 \pm 2.2$ & $4.7-16.7$ & $491.8 \pm 54.7$ & $341-611$ \\
Mild $(n=33)$ & $10.3 \pm 2.1$ & $4.7-15.3$ & $523.1 \pm 44.1$ & $442-611$ \\
Moderate $(n=19)$ & $9.7 \pm 2.4$ & $5.0-16.7$ & $487.0 \pm 52.4$ & $417-599$ \\
Severe $(n=41)$ & $9.0 \pm 2.1$ & $5.9-13.7$ & $470.2 \pm 53.9$ & $341-607$ \\
\hline
\end{tabular}

Data are the mean $\pm \mathrm{SD}$.

The difference in the slopes of the two regression equations are not statistically different based on the standard deviation of the slope. Using multivariant analysis to determine the hysteresis versus keratoconus severity trend after eliminating CCT effects, we found that hysteresis alone was not significant after removing CCT trends (severity versus CCT). Hysteresis, however, was a factor in addition to CCT, but its significance was just short of 0.05 .

The difference between the hysteresis of the mild and severe keratoconic eyes was significant $(P<0.01)$ but was not significant between the mild and moderate $(P>0.33)$ or between moderate and severe keratoconic eyes $(P>0.29$; unpaired $t$-test). The difference between the normal and mild keratoconus group was not significant $(P>0.29)$. The differences between the normal and moderate and the normal and severe groups were significant $(P<0.037$ and $P<0.0001$, respectively).

\section{Discussion}

Keratoconus generally starts at puberty and progresses until the third or fourth decade of life, ${ }^{3}$ after which it usually stabilizes. The exact etiology of keratoconus is not known, but it is more common in patients with the atopic conditions (42.2\%) hay fever, asthma, and atopic dermatitis, ${ }^{6}$ with a significantly increased level of $\operatorname{IgE}^{6-9}$; the endocrine diseases Addison's and hypothyroidism; connective tissue diseases such as Marfan's syndrome, Ehlers-Danlos syndrome, and osteogenesis imperfecta ${ }^{10}$; Down's syndrome ${ }^{1}$; and low economic status. ${ }^{11,12}$ A higher incidence of keratoconus is also associated with wearers of contact lenses, ${ }^{13}$ retinitis pigmentosa, aniridia, blue sclerosis, and Leber's amaurosis. ${ }^{1,10}$ Certain enzyme defi- ciencies, a reduced mechanical strength of the cornea, high collagenolytic activities and disruption in cross-linking of the cornea have all been implicated in the etiology of keratoconus. ${ }^{14}$ It may also be accompanied by the generalized connective tissue disorder characterized by weakness of the collagen tissues. $^{15}$

Previous studies have suggested various reasons for the histopathologic changes in the corneal tissue. These include a decrease in the number of normal collagen fibers, anomalies in the keratocyte membrane, fragmentation of the corneal basal epithelial membrane, degenerative changes of basal epithelial cells, a disintegrated Bowman's layer, ${ }^{16,17}$ a decreased level of glucose-6-phophate dehydrogenase ${ }^{18}$ and decreased collagen and increased structural glycoprotein.

Various investigators have tried to measure the ocular rigidity (viscoelasticity) of keratoconic eyes to assess the pathologic processes affecting the corneal tissue,,$^{2,18-20}$ but these studies either have been performed in vitro (on whole eye or excised corneal tissue) or have involved complicated mathematical calculations. Edmund ${ }^{2}$ investigated the viscoelasticity of the cornea by measuring the radius of the central corneal curvature, the coefficient of radius variation, the CCT, and the coefficient of thickness variation. He compared the viscoelasticity of keratoconic eyes and normal eyes, found that the distensibility of eyes was higher in normal eyes, and concluded that the increase in the distensibility may be an important factor in the pathogenesis of keratoconus. Brooks et al. ${ }^{21}$ investigated the ocular rigidity in 85 keratoconic eyes. They calculated the ocular rigidity coefficient from the combination of applanation tonometry and impression tonometry (Schiotz tonometer) using the Friedenwald ${ }^{22}$ nomogram and the line of best fit. They found that the ocular rigidity of the keratoconic

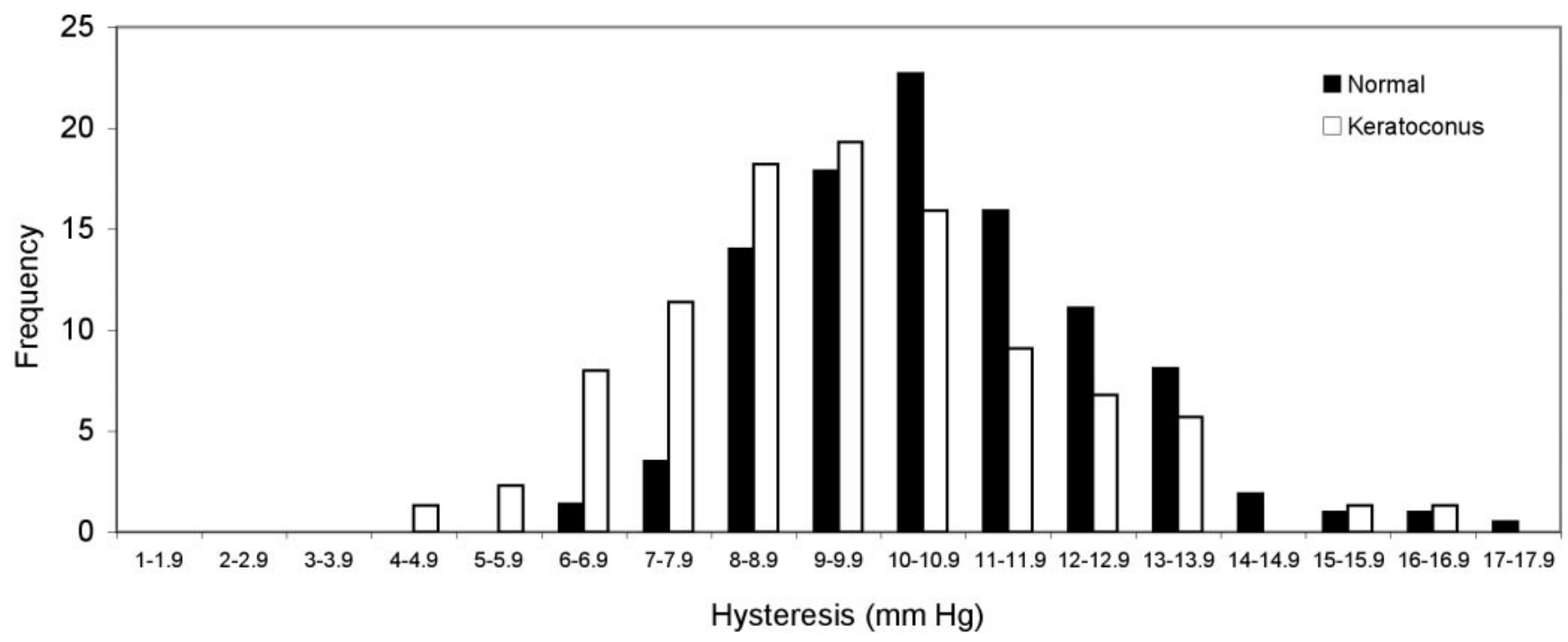

FIGURE 2. Histogram of hysteresis for normal and keratoconic eyes. 

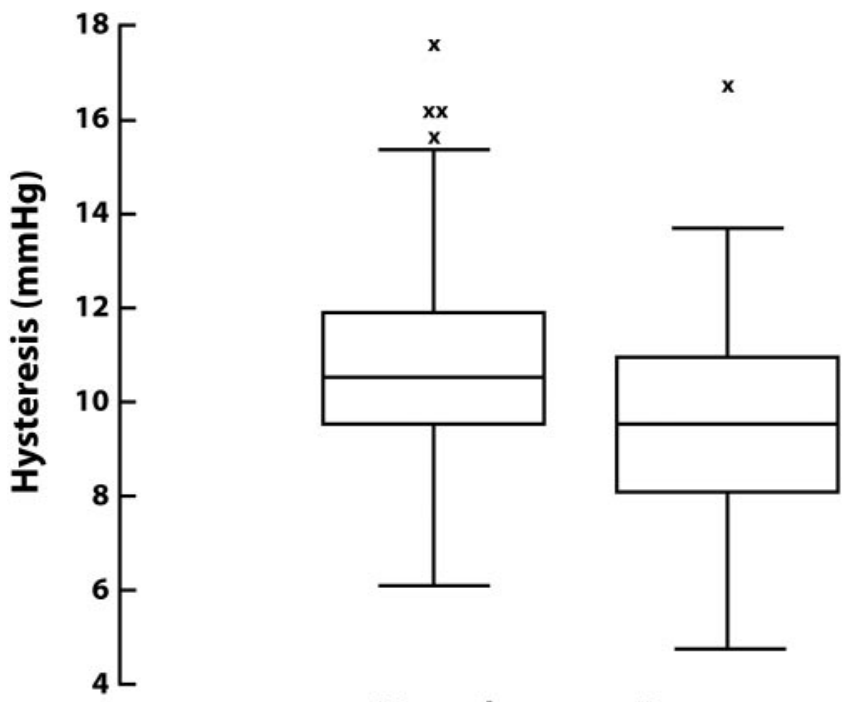

Normal

Keratoconus

FIGURE 3. Box-and-whisker plots (median and interquartile range) of hysteresis in normal and keratoconic eyes.

eyes was significantly lower than the control $(r=0.39, P<$ 0.001 ) when corneal thinning of $40 \%$ or more was present. They suggested that the corneal viscoelasticity is affected in keratoconic eyes.

Foster and Yamamoto ${ }^{18}$ also calculated the ocular rigidity coefficient by the Friedenwald ${ }^{22}$ nomogram on 84 keratoconic eyes and failed to demonstrate any statistically significant difference in corneal rigidity between normal subjects and patients with keratoconic eyes, unless corneal thinning was $60 \%$ or more. They were of the opinion that the Friedenwald method of calculating ocular rigidity was not accurate and did not reflect the true viscoelastic properties of keratoconic eyes. Hartstein and Becker ${ }^{20}$ also calculated rigidity by the Friedenwald $^{22}$ nomogram and found lower corneal rigidity in keratoconic eyes (0.010) in comparison to normal eyes (0.024). Edmund $^{19}$ in another study reported the corneal rigidity to be lower in patients with keratoconus eyes compared with the normal subjects and concluded that a decrease in the corneal matrix and a decrease in corneal tissue mass may be an important pathogenic factor in the development of keratoconus. Andreassen et al. ${ }^{23}$ also found that the corneal tissue in the keratoconic eye was more elastic than that in the normal subjects.

Moses $^{24}$ was of the opinion that the corneal shape (geometry) did not influence corneal rigidity. Nash et al. ${ }^{25}$ could not find any difference in the corneal viscoelasticity between normal and keratoconic eyes at the physiological IOP level of up to $30 \mathrm{~mm} \mathrm{Hg}$. In general, all these studies showed a reduced rigidity in keratoconic eyes, but all involved complicated mathematical calculations that are impractical for clinicians.

In this study, we measured hysteresis, a measure of ocular rigidity (viscoelasticity), using the ORA (Reichert). Measuring corneal biomechanical properties by applanation of force to the cornea requires a procedure capable of separating the contributions of the corneal resistance and the IOP, because the corneal resistance and true IOP are basically independent. The ORA releases a precisely metered air pulse that causes the cornea to move inward; thus, the cornea passes through applanation-inward applanation-and then the past applanation phase at which point its shape becomes slightly concave. Milliseconds after applanation, the air puff shuts off, resulting in a pressure decrease in a symmetrical fashion. During this phase, the corneal shape tries to gain its normal shape and the cornea again passes through an applanation phase- outward applanation. Theoretically, these two pressures should be the same, but this is not the case and this is described as the dynamic corneal response, which is said to be the resistance to applanation manifested by the corneal tissue due to its viscoelastic properties. The difference between the outward and inward pressures is termed hysteresis and is measured in millimeters of mercury.

The cornea reacts to stress as a viscoelastic material; for a given stress, the resultant corneal strain is time dependent. The viscoelastic response consists of immediate deformation followed by a rather slow deformation. ${ }^{19}$ The immediate elastic response of the ocular tunics seems to reflect the immediate elastic properties of the collagen fibers, and the steady state elastic response reflects the properties of the corneal matrix. ${ }^{19}$ The two applanation pressure readings inward and outward, are perhaps the result of an immediate elastic response and delayed or steady state elastic response, respectively, of the corneal tissue.

The results of the study show that the hysteresis in normal eyes was higher than that in keratoconic eyes. The mean hysteresis was $10.7 \mathrm{~mm} \mathrm{Hg}$ in normal eyes compared with 9.6 $\mathrm{mm} \mathrm{Hg}$ in keratoconic eyes. The difference was statistically significant $(P<0.0001$, unpaired $t$-test). The hysteresis data showed a wide range of values, all were within the normal range for the ORA. The histogram showed that the range of hysteresis in both normal and keratoconic eyes is between 7 and $13 \mathrm{~mm} \mathrm{Hg}$. The box-and-whisker plots show the median and interquartile range of the hysteresis and CCT of normal and keratoconic eyes, and these demonstrate the differences between the normal and keratoconic eyes. An analysis of a possible relationship between the CCT and hysteresis of normal and keratoconic eyes was performed (Figs. 5, 6). When a simple regression line was applied, it revealed a relationship showing a positive effect (i.e., the higher the CCT, the higher the hysteresis and vice versa). However, the correlation coefficient was poor (coefficient correlation, $r=0.45$ ), implying that hysteresis and CCT are related but are not measurements of the same biomechanical parameter. In the absence of another reliable measure of viscoelasticity, it is difficult to assess to what extent the hysteresis values are thickness (CCT) dependent rather than viscoelasticity dependent. It is, however, the feeling of Reichert that hysteresis is primarily viscoelastic dependent (Luce D, personal communication, 2005).
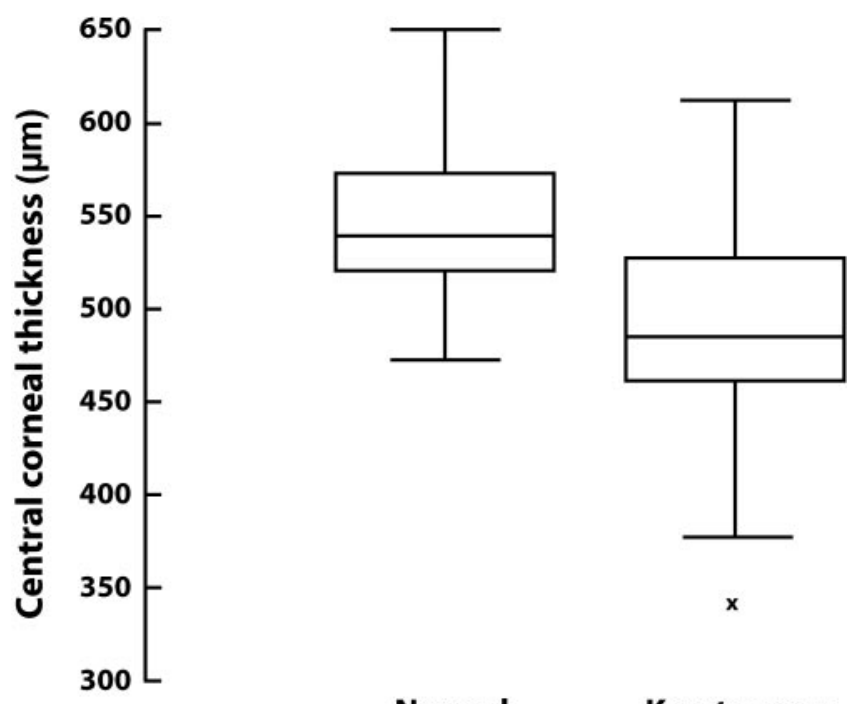

Normal

Keratoconus

Figure 4. Box-and-whisker plots (median and interquartile range) of CCT in normal and keratoconic eyes. 


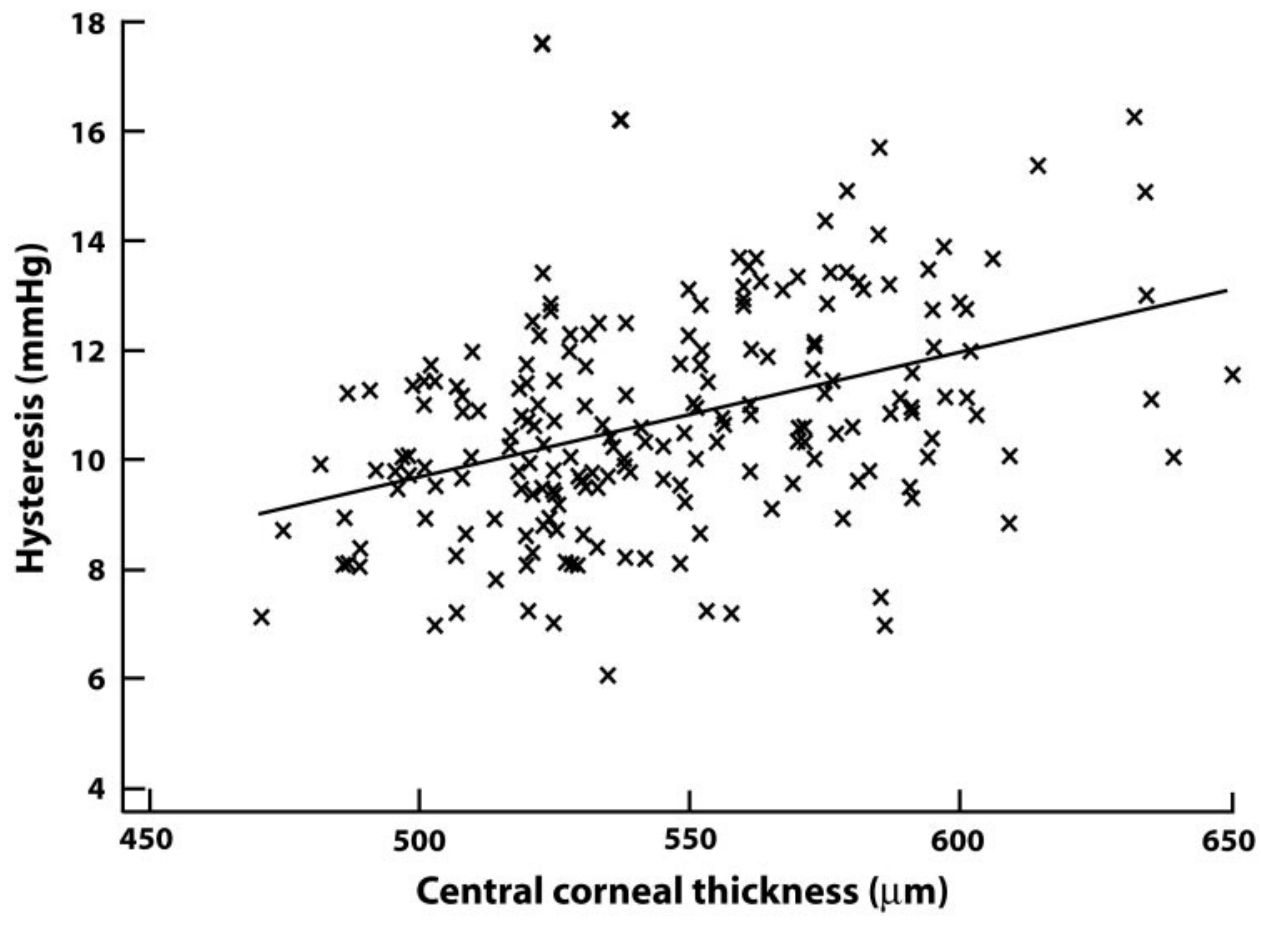

FiguRE 5. Scatterplot of relationship between hysteresis and CCT in normal eyes.
An analysis of hysteresis related to age and gender was performed in both normal and keratoconic eyes, and no correlation was found between age and hysteresis. The regression line in scatterplots (data not shown) was flat $(P>0.9)$, and also no correlation was found between hysteresis in males and females.

A further analysis of the keratoconic eyes was performed by grading the keratoconus as mild, moderate, and severe on the basis of Orbscan II (Bausch \& Lomb Surgical) readings and clinical grading of the Orbscan image. The new grading scale for the severity of the keratoconus based on Orbscan II, is presented in the Methods section. The analysis revealed decreasing hysteresis values with the severity of the disease. Mean hysteresis in the mild keratoconic eyes was $10.3 \mathrm{~mm} \mathrm{Hg}$; in moderately affected eyes, 9.7; and in severely affected eyes, $9.0 \mathrm{~mm} \mathrm{Hg}$. Both hysteresis and CCT were different in each of the mild, moderate, and severe groups and the difference between mild and severe groups was statistically significant. This finding demonstrates that hysteresis declines as the keratoconus becomes more severe. However, this technique cannot differentiate normal corneas from mild keratoconus. Further studies are needed to assess whether this is because

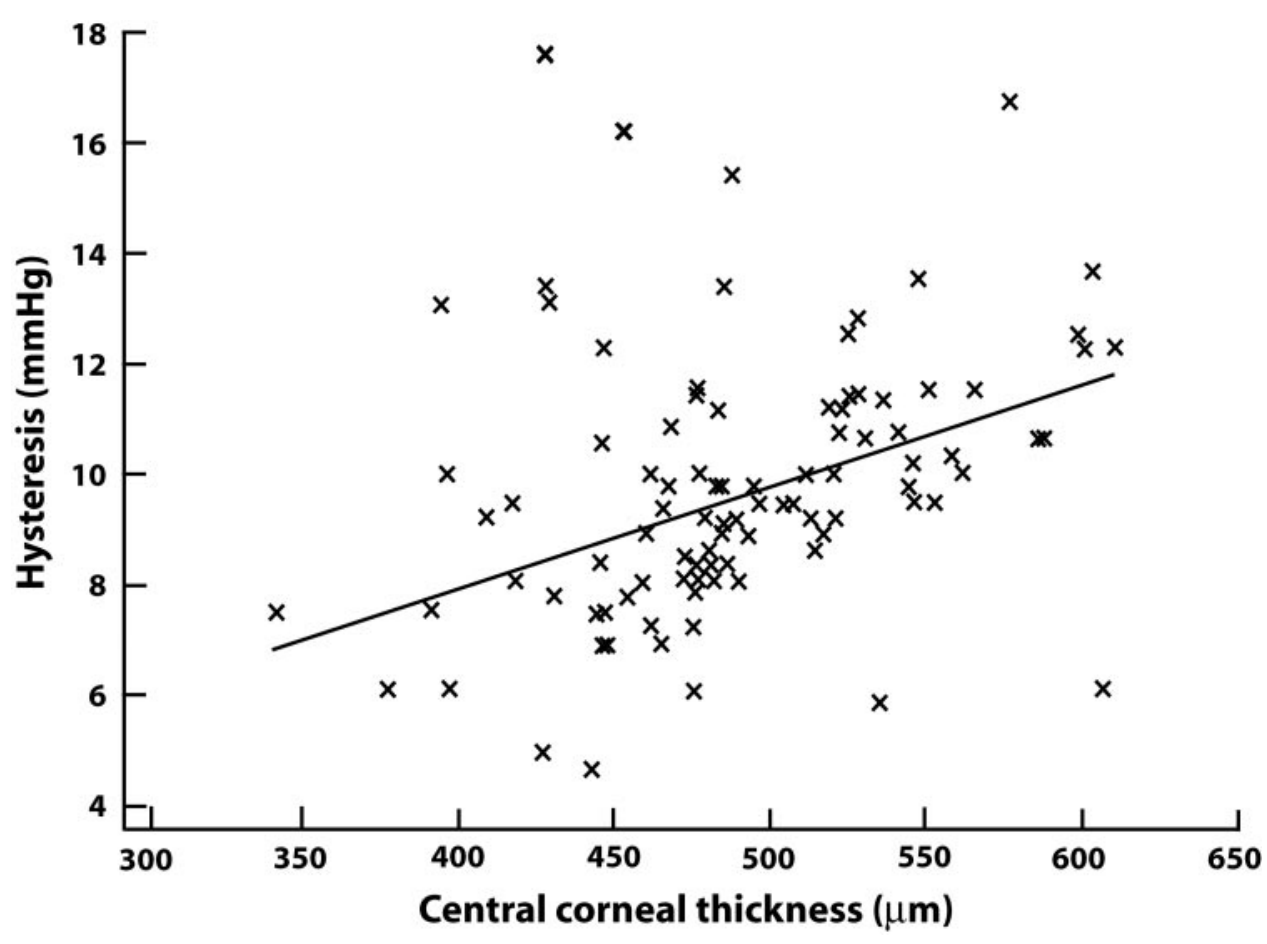

FiguRE 6. Scatterplot of relationship between hysteresis and CCT in keratoconic eyes. 
hysteresis itself is not affected in mild keratoconus or whether the technique used to distinguish keratoconus as "mild" could be improved.

The overall analysis of our data showed that corneal hysteresis in the normal eyes was higher than that in the keratoconic eyes. Although many studies have been reported on the ocular (corneal) rigidity in keratoconic eyes versus normal eyes, this is the first study performed to investigate the relationship between hysteresis in normal and keratoconic eyes with a new technique using the ORA (however, part of this data set has been reported ${ }^{4}$ ). This result is in agreement with previous studies performed to find the ocular rigidity (viscoelasticity) in normal and keratoconic eyes. ${ }^{19-21,23}$ The recently published paper by Luce ${ }^{4}$ (the first reported study to be performed on the same instrument) included a cohort of patients from this study and agreed with our findings that hysteresis in keratoconic eyes was lower than in normal eyes. However, the data published by Luce (based on a poster presentation by this group at the American Academy of Ophthalmology, New Orleans, Louisiana) reports on the Generation 1 of the ORA. This study is based on Generation 3 software for the ORA and hence there are some numerical differences between the two reports.

No variation in the slopes was found for CCT versus hysteresis in the normal and keratoconic groups. Multivariant analysis showed that CCT was the predominant factor for severity, although there was an effect of hysteresis (which was almost significant). Further work is required to assess the importance of these two factors.

Hysteresis is a parameter to characterize the biomechanical status of the cornea, but a clear separation of normal and keratoconic corneas is not possible because the ranges for hysteresis overlap because of interindividual variations. Hysteresis is likely to be a useful additional measurement to assess ocular rigidity. The ORA may also be useful to assess progression of disease, as hysteresis may change before topographic or clinical changes becoming apparent. This may make the ORA useful to help decide likely outcomes with keratoconus such as the chance of proceeding to keratoplasty.

\section{References}

1. Rabinowitz YS. Keratoconus (major review). Surv Opbthalmol. 1998;42:297-319.

2. Edmund C. Assessment of an elastic model in the pathogenesis of keratoconus. Acta Ophthalmol. 1987;65:545-550.

3. Li X, Rabinowitz YS, Rasheed K, Yang H. Longitudinal study of the normal eyes in unilateral keratoconus patients. Ophthalmology. 2004; 111:440 - 446 .

4. Luce DA. Determining in-vivo biomechanical properties of the cornea with an ocular response analyzer $J$ Refract Cataract Surg. 2005;31:156-162.
5. Zar JH. Biostatistical Analysis. 2nd ed. Englewood Cliffs, NJ: Prentice-Hall; 1984.

6. Davies PD, Lobascher D, Menon JA, Rahi AH, Ruben M. Immunological studies in keratoconus. Trans Opbthalmol Soc UK. 1976; 96:173-178.

7. Swann PG, Waldron HE. Keratoconus: a clinical spectrum. $J$ Am Optom Assoc. 1986;57:204-209.

8. Kemp EG, Lewis CJ. Immunoglobulin patterns in keratoconus with particular reference to total and specific IgE levels. Br J Ophthalmol. 1982;66:717-720.

9. Kemp EG, Lewis CJ. Measurement of total and specific IgE levels in the management of a family exhibiting a high incidence of keratoconus. Acta Opbthalmol. 1984;62:524-529.

10. Duke-Elder S, Leigh AG. Diseases of the Outer Eye. London: Kimpton; 1964. Systems of Opbthalmology; Vol 8, Part 2.

11. Karseras AG, Ruben M. Aetiology of keratoconus. Br J Ophthalmol. 1976;60:523-525.

12. Woodward EG. Keratoconus: maternal age and social class. $\mathrm{Br} J$ Opbthalmol. 1981;65:104-107.

13. Hartstein J. Corneal warping due to wearing of corneal contact lens; a report of 12 cases. Am J Opbthalmol. 1965;60:1103-1104.

14. Krachmer JH, Feder RS, Belin MW. Keratoconus and related noninflammatory corneal thinning disorders. Surv Opbthalmol. 1984; 28:293-332.

15. Robertson I. A new aspect of keratoconus. Aust J Opbthalmol. 1974;2:144-147.

16. Chi HH, Katzin HM, Teng CC. Histopathology of keratoconus. Am J Opbthalmol. 1956;42:847-860.

17. Teng C. Electron microscope study of the pathology of keratoconus. Am J Opbthalmol. 1963;55:18-47.

18. Foster CS, Yamamoto GK. Ocular rigidity in keratoconus. Am J Ophthalmol. 1978;86:802-806.

19. Edmund C. Corneal rigidity and ocular rigidity in normal and keratoconic eyes. Acta Ophthalmol. 1988;66:134-140.

20. Hartstein J, Becker B. Research into the pathogenesis of keratoconus: a new syndrome: low ocular rigidity, contact lenses and keratoconus. Arch Ophthalmol. 1970;84:728-729.

21. Brooks AMV, Robertosn IF, Mahoney AM. Ocular rigidity and intraocular pressure in keratoconus. Aust J Opbthalmol. 1984;12: 317-324.

22. Friedenwald JS. Contribution to the theory and practice of tonometry. Am J Opbthalmol. 1937;20:985-1024.

23. Andreassen TT, Simonsen AH, Oxlund H. Biomechanical properties of keratoconus and normal corneas. Exp Eye Res. 1980;31: 435-441.

24. Moses RA. Theory and calibration of the Shiotz tonometer; VA mathematical model of Shiotz tonometry - the volume of indentation. Invest Ophthalmol. 1971;10:601-604.

25. Nash IS, Greene PR, Foster CS. Comparison of mechanical properties of keratoconus and normal corneas. Exp Eye Res. 1982;35: 413-423. 